Med Klin Intensivmed Notfmed 2013.

108:182-183

DOI 10.1007/s00063-012-0161-7

Online publiziert: 28. Februar 2013

(c) Springer-Verlag Berlin Heidelberg 2013

\author{
M. Kochanek ${ }^{1} \cdot$ T. Staudinger ${ }^{2}$ \\ ${ }^{1}$ Klinik I für Innere Medizin und Centrum für Integrierte Onkologie, \\ Internistische Intensivstation, Klinikum der Universität zu Köln \\ ${ }^{2}$ Allgemeines Krankenhaus der Stadt Wien, Universitätsklinik für Innere \\ Medizin I, Intensivstation 13.i2, Medizinische Universität Wien
}

\title{
Der onkologische Patient in der Intensivmedizin
}

ziehen. Dazu kommt, dass aufgrund der rasanten Entwicklung der antineoplastischen Therapien und der damit einhergehenden Verbesserung der Prognose der Druck auf die Intensivmedizin steigt, Patienten mit therapieassoziierten Komplikationen adäquat zu versorgen. Im Jahre 2006 betitelte Elie Azoulay aus der Pariser Arbeitsgruppe ein Editorial mit „The intensive care support of patients with malignancy: do everything that can be done" [2]. Hat sich in den dazwischen liegenden Jahren die Situation wirklich grundlegend geändert und, wenn ja, warum?

Eine Reihe von Erkenntnissen hat sicherlich dazu beigetragen, das Bild des kritisch kranken Krebspatienten zu modifizieren:

- Die Lebenserwartung und die Prävalenz von Krebserkrankungen steigen stetig an, was unweigerlich zu einer Zunahme der Zahl kritisch erkrankter Krebspatienten führt. Bei 15-20\% aller ICU-Patienten liegt eine Krebserkrankung vor, d. h. jeder siebente bis fünfte Patient einer Europäischen Intensivstation leidet an einer malignen Grunderkrankung [3, 4]. Die Auseinandersetzung mit diesem speziellen Patientenkollektiv liegt deshalb für Intensivmediziner nahe.

- Noch vor ein bis zwei Jahrzehnten lag das ICU-Überleben von Krebspatienten mit Zwei- und Mehrorganversagen bei 10-20\%. In der Zwischenzeit konnte eine deutliche Verbesserung der Prognose erreicht werden. Einzelne spezialisierte Zentren berichten mittlerweile sogar ICU-Überlebensraten von knapp 70\% bei Patienten mit invasiver Beatmung und Mehrorganversagen [5]. Diese Entwicklung ist einerseits der verbesserten Patientenselektion geschuldet, andererseits haben Fortschritte im Management von Organdysfunktionen, ein erweitertes Wissen um die Diagnose und Therapie spezifischer Komplikationen sowie neue onkologische und infektiologische Therapieoptionen zu einer Verbesserung der Prognose beigetragen.

- Die Therapieoptionen für viele Krebsarten haben sich vervielfältigt. Die Situation, dass keine weitere Behandlungsmöglichkeit mit der Chance auf lebensverlängernde Wirksamkeit besteht, ist dementsprechend seltener geworden.

- Neue Erkenntnisse zum spezifischen therapeutischen Management häufig zu einem Aufenthalt auf der Intensivstation führender akuter Krisen helfen Komplikationen zu vermeiden und so das ICU-Überleben $\mathrm{zu}$ verbessern. Insbesondere seien hier das respiratorische Versagen [6] und schwere Infektionen bzw. Sepsis und septischer Schock [7] erwähnt. Beide Syndrome bringen in dieser Patientengruppe sowohl prognostisch, diagnostisch, als auch therapeutisch etliche wichtige Besonderheiten mit sich. Die erfolgreiche Versorgung von Krebspatienten auf einer Intensivstation setzt spezifisches Wissen der Intensivmediziner und eine gute Zusammenarbeit mit Hämatologen und Onkologen voraus.

- Etliche „traditionelle“ Prognosefaktoren, z. B. das Vorliegen einer 
Neutropenie bei Sepsis, eine rezente Chemotherapie oder eine stattgehabte autologe Blutstammzelltransplantation, haben keinen Einfluss auf das ICU-Überleben. Letzteres hängt grundsätzlich ab von Alter, Komorbiditäten, Performance-Status und dem Ausmaß der kritischen Erkrankung bzw. der Organdysfunktionen, nicht jedoch von den Charakteristika der malignen Grunderkrankung. Diese bestimmen jedoch den Langzeitverlauf nach einem Intensivaufenthalt und müssen bei der Entscheidung für oder gegen eine ICU-Aufnahme berücksichtigt werden.

Alle erwähnten Faktoren tragen sicherlich dazu bei, die nach wie vor bestehende Skepsis vieler Intensivmediziner gegenüber der Aufnahme von Patienten mit maligner Grundkrankheit als ungerechtfertigt erscheinen zu lassen. Dennoch erscheint die erwähnte Forderung „Do everything that can be done" etwas überzogen, da nach wie vor viele offene Fragen bestehen:

- Wann ist der optimale Zeitpunkt einer Übernahme?

- Sollten kritisch kranke onkologische Patienten nur an spezialisierten Zentren behandelt werden?

- Wer kommt infrage für nichtinvasive Beatmung bzw. ist Intubationsvermeidung immer das Ziel beim Management des respiratorischen Versagens?

- Wie kann die nach wie vor unbefriedigende Prognose kritisch kranker Patienten in den Frühphasen nach Stammzelltransplantation verbessert werden?

- Welche Patienten sollen nicht auf die Intensivstation?

- Wann und wie soll eine Therapie begrenzt oder abgebrochen werden?

- Wie geht man mit einer akuten Krise onkologischer Patienten im palliativen Setting um?

- Wie kann der Wille des Patienten besser berücksichtigt werden?

Das vorliegende Themenheft wird versuchen, die derzeit bestehende Evidenz und Erkenntnis zusammenzufassen und manche dieser Fragen zu beantworten. Umfangreiche weitere Forschung, Erfah- rungsaustausch und Meinungsbildung werden jedoch erforderlich sein, um jeden Patienten einem individuell adäquaten und sowohl im medizinischen Sinne als auch im Hinblick auf die Lebensqualität und auf die zur Verfügung stehenden Ressourcen erfolgreichen Management zuzuführen. Die Etablierung spezifischer Arbeitsgruppen innerhalb der Deutschen Gesellschaft für Internistische Intensivmedizin (DGIIN) und der Österreichischen Gesellschaft für Internistische und Allgemeine Intensivmedizin und Notfallmedizin (ÖGIAIN) trägt diesen Forderungen Rechnung. Interessierte sind herzlich zu Kontaktaufnahme über die Gesellschaften eingeladen.

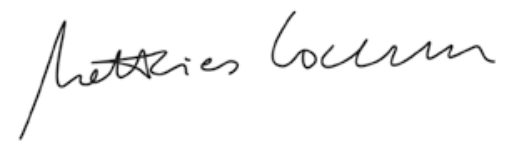

Dr. Matthias Kochanek

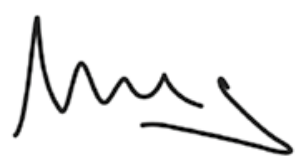

Professor Dr. Thomas Staudinger

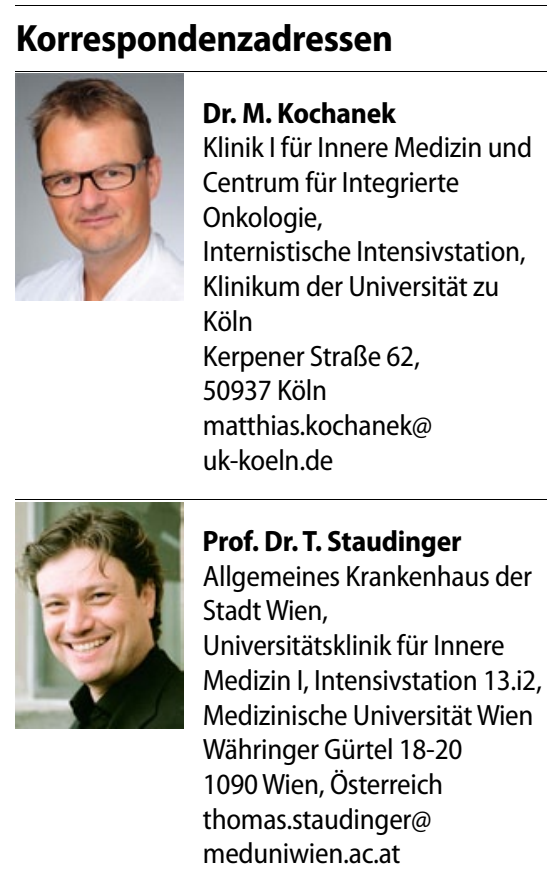

Interessenkonflikt. Der korrespondierende Autor gibt für sich und seine Koautoren an, dass kein Interessenkonflikt besteht.

\section{Literatur}

1. Sculier JP (1991) Intensive care in the treatment of cancer patients. Curr Opin Oncol 3: 656-662

2. Azoulay E, Afessa B (2006) The intensive care support of patients with malignancy: do everything that can be done. Intensive Care Med 32: 3-5

3. Taccone FS, Artigas AA, Sprung CL, et al (2009) Characteristics and outcomes of cancer patients in European ICUs. Crit Care 13: R15

4. Soares M, Caruso P, Silva E, et al (2010) Characteristics and outcomes of patients with cancer requiring admission to intensive care units: a prospective multicenter study. Crit Care Med 38: 9-15

5. Lecuyer L, Chevret S, Thiery G et al (2007) The ICU trial: a new admission policy for cancer patients requiring mechanical ventilation. Crit Care Med 35: 808-814

6. Molina R, Bernal T, Borges et al (2012) Ventilatory support in critically ill hematology patients with respiratory failure. Critical Care 16: R133

7. Pène F, Percheron S, Lemiale V, et al (2008) Temporal changes in management and outcome of septic shock in patients with malignancies in the intensive care unit. Crit Care Med 36: 690-696 